\title{
Study on Mass Transfer Characteristics of Hydrate-based Gas Absorber
}

\author{
Shun TAKANO*, Ryosuke EzUre, Yusuke TAKAHASHI, Hiroyuki Komatsu, Kazuaki Yamagiwa, and Hideo TAJIMA* \\ Graduate School of Science and Technology, Niigata University, 2-8050 Ikarashi, Niigata 950-2181, Japan
}

\begin{abstract}
Hydrated-based gas separation is a method capable of selectively separating and recovering greenhouse gases. Although a conventional hydrate-based gas separation apparatus is a batch or a semi-batch system, continuous operation is preferable to increase the throughput of gas without changing the apparatus volume. Recently, we proposed a flow type apparatus to allow continuous operation of hydrate formation (absorption) and subsequent decomposition (desorption). The aim of this study is to investigate the mass transfer characteristics of the continuous apparatus using the HFC134a- $\mathrm{N}_{2}$ mixed gas system. The volumetric mass transfer coefficient was calculated especially during a steady state of gas absorption. Besides, we compared mass transfer performance between the hydrate-based gas absorber and a conventional bubble column. Sodium dodecyl sulfate was used as a hydrate dispersant. In the flow type apparatus, the gas-liquid contact was good and the hydrate slurry state was observed during hydrate formation. In the surfactant solution, the volumetric mass transfer coefficient increased in comparison with that in water. The volumetric mass transfer coefficient with hydrate was higher than that of the bubble column. These results suggest that hydrate formation improves gas absorption performance.
\end{abstract}

\section{Introduction}

Currently, global warming is becoming serious, and it is necessary to separate and recover the greenhouse gases that are the cause. As a solution to this problem, separation methods using gas hydrate have attracted attention. The hydrated-based gas separation is a method of selectively separating specific gases using the following gas thermodynamic conditions; if a large difference in the hydrate formation conditions is observed among guest gases, the component of which the hydrate formation conditions are milder (lower pressure and higher temperature) could be enriched in the hydrate phase. This method has advantages of low costs of material and safety because separation media is water only. To design a separation apparatus, mass transfer characteristics are required, but the hydrate formation process is complicated, and there is little information on mass transfer. In previous studies, we have considered a gas-liquid-hydrate three-phase as a gas-hydrate slurry pseudo two-phase, it was found that the apparent volumetric mass transfer coefficient was mainly affected by the amount of formed hydrate particle and the pattern of pseudo gas-liquid flow (Shimizu et al., 2014). Although we have used a flow type apparatus in semi-batch operation, continuous operation is preferable to increase the throughput of gas without changing the apparatus volume. So we proposed a flow type apparatus to allow continuous operation of hydrate formation (absorption) and subsequent decomposition (desorption). This study presents mass transfer characteristics to evaluate the performance of the flow type apparatus using the HFC134a- $\mathrm{N}_{2}$ mixed gas system as a lowpressure model of greenhouse gases. The apparent volumetric mass transfer coefficient was calculated especially during a steady state of gas absorption. Besides, we investigated the effects of hydrate particles and flow behavior on the volumetric mass transfer coefficient and compared mass transfer performance between the hydrate-based gas absorber and a conventional bubble column.

\section{Experimental Section}

\subsection{Hydrate formation and decomposition}

\subsubsection{Experimental apparatus}

In the experiment, the hydrate gas absorber (flow type) shown in Figure 1. In the reactor, Kenics-type mixing elements of SUS316 static mixer (Noritake Co. Ltd., Japan, 24 elements, pitch 1.5) as the flow reactor was inserted into a glass tube (400 mm length, i.d. $11 \mathrm{~mm})$. In another chamber, a glass recovery vessel with an internal volume of $500 \mathrm{~mL}$ and a glass tube $(500 \mathrm{~mm}$ length, i.d. $20 \mathrm{~mm}$ ) for observing gas bubbles and hydrate particles at the time of hydrate decomposition are installed. The hydrate formation and decomposition apparatuses are

Corresponding Authors: f19b058d@mail.cc.niigata-u.ac.jp (S.Takano), h_tajima@eng.niigata-u.ac.jp (H. Tajima) 
connected by a flexible tube, and the hydrate formed in the reactor is sent to the recovery vessel by the flow of water and gravity. A mass flow controller (MFC) was used to measure the inlet gas flow rate, and a mass flow meter (MFM) (KOFLOC) was used to measure the outlet gas flow rate. The gas flow rates of HFC134a gas and $\mathrm{N}_{2}$ gas were calibrated using a high precision soap film flowmeter (HORIBA, SF-2V). Experimental pressure in the reactor was controlled by a back pressure valve (AP tech) and measured by a pressure transducer (Nagano, KH-15). Hydrate formation and decomposition were carried out in separate thermostatic chamber (EYELA, and Fukushima Kogyo Co. Ltd., Japan). The temperatures inside the reactor and in the recovery vessel were measured using a Pt temperature measuring resistor. The pressure and temperature in the reactor, the outlet gas flow rate, and the temperature in the recovery vessel were recorded every second using a data logger system (KEYENCE, NR1000), and the data were collected on a connected PC.

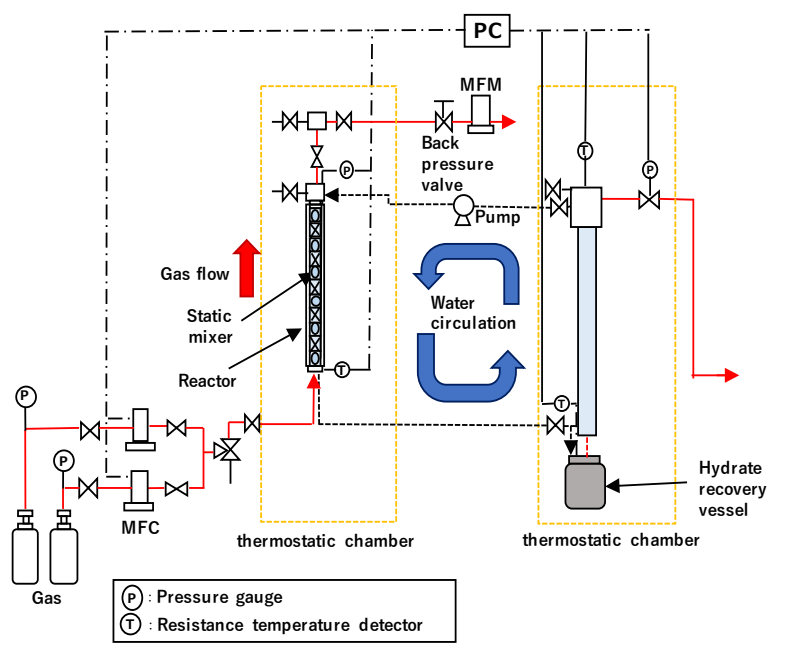

Figure 1. Hydrate gas absorber (flow type)

\subsubsection{Experimental condition}

In the experiment, a mixed system of HFC134a (99.9\%, Mitsui-Dupont Fluorochemicals Co., Ltd.) and $\mathrm{N}_{2}$ (99.9\%, Niigata IGS Co., Ltd.) was used as a lowpressure model gas of greenhouse gases. Each gas was mixed through the MFC and supplied. The total amount of mixed gas was introduced into the apparatus at $200 \pm$ $2 \mathrm{~mL} / \mathrm{min}$. Deionized water was used in the hydrate formation experiments, and was brought into countercurrent contact with the gas at $109.6 \mathrm{~mL} / \mathrm{min}$. The measurement with MFM and MFC was performed with reference to atmospheric pressure and room temperature, and the experimental pressure was set to $0.325 \pm 0.01 \mathrm{MPa}$, and the experimental temperature was set to $278 \pm 1 \mathrm{~K}$.

\subsubsection{Experimental procedure}

The inside of the apparatus was degassed by a vacuum pump (SATO VAC INC., Japan). Deionized water was charged into the apparatus and cooled to the desired temperature. During cooling, the water supply pump was operated to circulate water. The gas mixture was then injected into the reactor at the desired pressure, and the gas mixture was brought into countercurrent contact with water to form a hydrate. The composition of the initial gas mixture was controlled by each gas flow rate. When the change in the flow rate becomes constant, it was judged that the water in the inside of the reactor reached saturation. The inlet gas was then recovered in the gas bag from the outlet of the MFM. The inlet gas flow rate $F_{\text {in }}\left[\mathrm{m}^{3} / \mathrm{s}\right]$ and the mole fraction $y_{\text {in }}[-]$ of the HFC134a of the inlet gas were determined respectively. As the hydrate formation started, the outlet gas flow rate gradually decreased. The outlet gas flow was constant while the hydrate formation was kept steady. This gas flow rate was defined as an outlet gas flow rate $F_{\text {out }}$ $\left[\mathrm{m}^{3} / \mathrm{s}\right]$. Thereafter, the gas discharged from the MFM was collected by the gas bag. The component of the recovered gas was defined as the mole fraction $y_{\text {out }}[-]$ of HFC134a of the outlet gas. The hydrate formed for about 30 minutes, and a high-performance camera was used to take pictures of the flow appearance in the reactor as appropriate. The mass transfer rate $N_{A}$ [mol/s] of HFC134a to the hydrate slurry solution was calculated from the difference in inlet and outlet gas flow rates and the component mole fraction. After hydrate formation, hydrate particles, experimental pressure and temperature were maintained in the recovery vessel. The recovered hydrate dissociated by releasing the pressure and increasing temperature and gases generated from hydrate slurry were recovered in a gas bag. All gas samples were analyzed by gas chromatograph. Sodium dodecyl sulfate (SDS) was used as a dispersant. The SDS solution was prepared by measuring 2 liters of deionized water with a graduated cylinder and adding sodium dodecyl sulfate (95.5\%, FUJIFILM Wako Pure Chemical Corporation) measured with an electronic balance so that the desired concentration was achieved therein. The SDS solution was introduced into a hydrate-based gas absorber and experiments were conducted in the same way as the case of using deionized water.

\subsection{Gas absorption in bubble column}

In the experiment, the HFC134a- $\mathrm{N}_{2}$ mixed gas system used is blended to the desired concentration and the feed concentration is previously prepared in the cylinder by manufacture. The bubble column is made of acrylic and has an inner diameter of $106 \mathrm{~mm}$ and a height of $880 \mathrm{~mm}$. Deionized water was pumped from the tank, and was allowed to flow from the top of the column. The flow rate was adjusted using a water flow meter. After the liquid flow rate and the water-surface height became constant, the mixed gas was supplied from the bottom of the column through a glass filter (pores: 100 to $160 \mu \mathrm{m}$ ) into the bubble column for countercurrent contact. The inlet and outlet gas flow rate, liquid flow rate, and temperature in the column were measured and recorded every 4 minutes. When the outlet gas flow rates at the three observation points became the same, it was judged as steady state. After the outlet gas flow rate became steady, the outlet gas was collected five times in the gas 
bag. The feed gas was then recovered. The recovered gas was analyzed by gas chromatograph. The concentration of HFC134a in the outlet gas was averaged over five sample bags. The mass transfer rate $N_{A}$ of HFC134a to the water was calculated from the difference in inlet and outlet gas flow rates and the component mole fraction similar to hydrate formation experiments.

\section{Result and Discussion}

\subsection{Mass transfer rate of HFC134a}

The relationship between the HFC134a mole fraction in feed gas and the mass transfer rate $N_{A}$ in the hydratebased gas absorber is shown in Figure 2. In deionized water, $N_{A}$ increased linearly as $y_{i n}$ increased. This is because the amount of HFC134a contained in the hydrate slurry increases as $y_{\text {in }}$ increases, and the amount of hydrate formation increases. The $N_{A}$ using the SDS solution is also shown in Figure 2. $N_{A}$ was higher than that using the deionized water. The flow behavior during hydrate formation is shown in Figure 3. In both $y_{i n}=0.5$ and $y_{i n}=0.7$, the bubbles and hydrate particles using the SDS solution were small and dispersed. Because SDS acted as a dispersing agent, the gas-liquid contact area increased as a result of bubbles and hydrate particles dispersed regardless of $y_{i n}$.

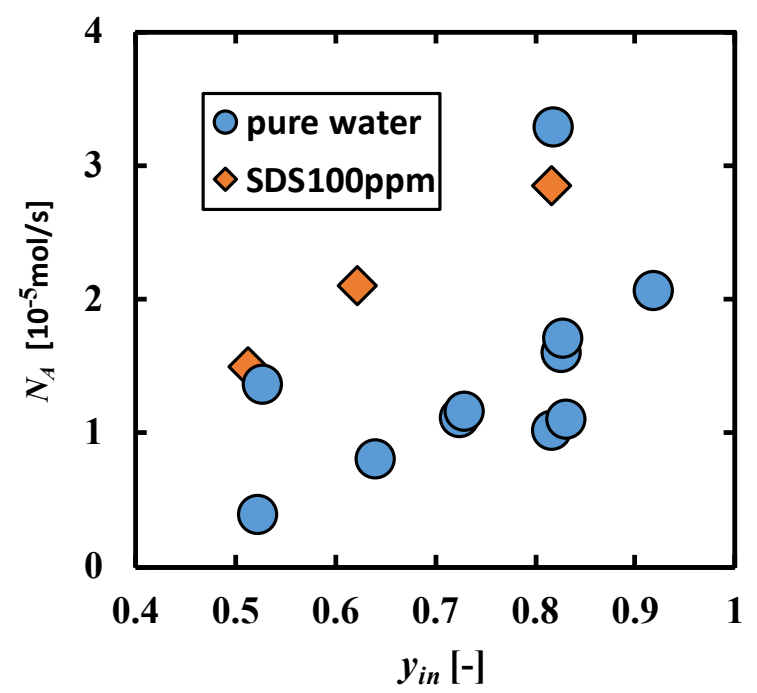

Figure 2. Relation of $y_{i n}$ and $N_{A}$

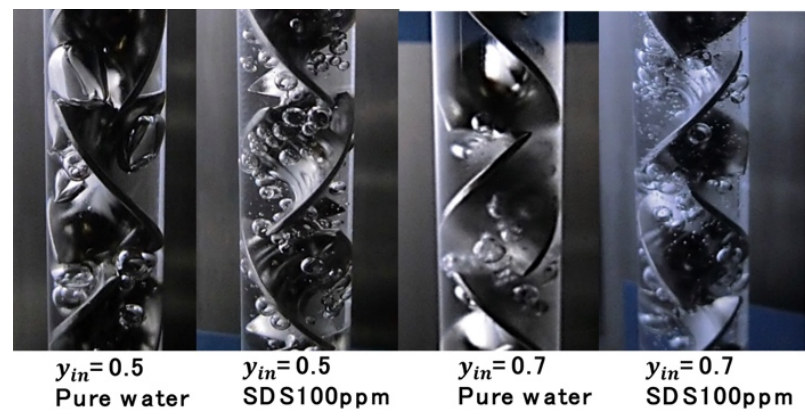

Figure 3. Comparison of flow behavior

\subsection{Volumetric mass transfer coefficient}

The gas absorption reaction in the experimental apparatus is very complex. In the reactor, gas dissolution, hydrate formation on bubble surface, hydrate formation in bulk solution, gas uptake into hydrate and gas desorption from hydrate will simultaneously occur. In this experiment, we have considered a gas-liquid-hydrate three-phase as a gas-hydrate slurry pseudo two-phase (Shimizu et al., 2014). It is assumed that overall hydrate formation consisted of hydrate formations in the gasliquid interface and in the bulk and desorption of hydrate did not occur (Tajima et al., 2010). Therefore, the absorption reactor will be organized into two stages: gas dissolution and overall hydrate formation. In this study, the following assumptions were also made for the calculation of apparent volumetric mass transfer coefficient: During hydrate formation, water is always saturated with gas mixture, the gas reduced in gas phase is consumed to hydrate formation only, and the reduction of water with hydrate formation is negligible because excess water was used. From the above assumption of gas absorption, the mass transfer rate of HFC134a to hydrate slurry solution, $N_{A}$, was expressed as follows (Sobieszuk and Pohorecki, 2010):

$$
\frac{N_{A}}{V_{R}}=K_{L} a \Delta C
$$

where $V_{R}$ is the volume of the reactor [ $\mathrm{m}^{3}$ ], $K_{L} a$ is overall liquid-side volumetric mass transfer coefficient of HFC134a $[1 / \mathrm{s}]$, and $\Delta C\left[\mathrm{~mol} / \mathrm{m}^{3}\right]$ is calculated as follows,

$$
\Delta C=C^{*}-C
$$

where $C^{*}$ is equilibrium concentration of HFC134a in slurry solution $\left[\mathrm{mol} / \mathrm{m}^{3}\right], C$ is concentration of $\mathrm{HFC} 134 \mathrm{a}$ in slurry solution $\left[\mathrm{mol} / \mathrm{m}^{3}\right]$. In this calculation, $\Delta C$ is used as the logarithmic mean of inlet liquid-side overall driving force, $\Delta C_{i n}$, and the logarithmic mean of outlet liquid-side overall driving force, $\Delta C_{\text {out }}$. Although it is difficult to measure the concentration of the HFC134a solution directly, we calculated $C^{*}$ and $C$ indirectly. $C^{*}$ can be calculated by determining the theoretical gas recovery rate from the $P$ (pressure)- $y$ (gas composition) equilibrium diagram of the HFC134a- $\mathrm{N}_{2}$ mixed gas hydrate (Nagata et al., 2009). $C$ can be considered as the sum of the saturated solubility of HFC134a in water and the concentration of HFC 134a absorbed within hydrate in the slurry. The saturated solubility of HFC134a in water can be calculated from Henry's law constant (Kavanaugh and Trussell, 1980) for now though a change in HFC134a concentration in water is negligible due to the assumption in which water is saturated during hydrate formation. The concentration of HFC134a absorbed within hydrate can be calculated from $N_{A}$. In this experiment, there was no formation of hydrate plug (that is a state in which gas bubbles are covered with hydrate because of the high hydrate formation rate), and the state of hydrate slurry was maintained. The relationship between the HFC134a mole fraction in feed 
gas $y_{i n}$ and the liquid side mass transfer coefficient $K_{L} a$ in the hydrate-based gas absorber is shown in Figure 4. In the deionized water, $K_{L} a$ (circles) showed an almost constant value, with an average of $0.0195 \mathrm{~s}^{-1}$ (standard deviation 0.0136). In the SDS solution, $K_{L} a$ (diamonds) was a higher value than that in the deionized water, and the average was $0.0486 \mathrm{~s}^{-1}$ (standard deviation 0.0130). This result indicates that SDS disperses gas bubbles and particles, and in particular, $K_{L} a$ is greatly affected by bubble size reduction. In other words, surface area $a$ becomes a large especially and therefore $K_{L} a$ shows high value.

\subsection{Comparison with bubble column}

Figure 4 also shows a comparison of $K_{L} a$ in the hydrate gas absorber and bubble column. Although the HFC134a mole fraction in feed gas and temperature conditions do not match because hydrate stability conditions must be taken into consideration, $K_{L} a$ of hydrate absorber was about 10 times higher than that of the bubble column (triangles, and rectangles) when the HFC134a- $\mathrm{N}_{2}$ mixed gas was used. $K_{L} a$ showed a higher value also when the SDS solution was used in the bubble column. However, it was lower than the value of the hydrate gas absorber. From this, it can be said that the hydrate gas absorber is better as a gas separation apparatus.

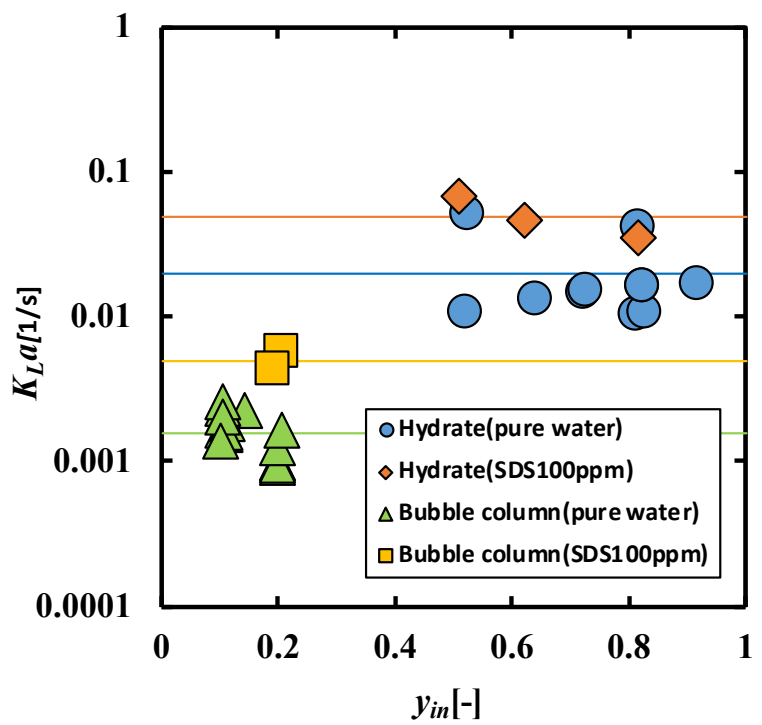

Figure 4. Comparison with $K_{L} a$ in bubble column

\section{Conclusion}

The performance of the hydrated-based gas absorber was evaluated by the volumetric mass transfer coefficient, and the gas absorption experiment by the bubble column was performed as a control experiment to compare the performance. We could calculate $K_{L} a$ of the hydratebased gas absorber, according to several assumptions for simplification. In the SDS solution, $K_{L} a$ increased due to the increase in the gas-liquid contact area. The $K_{L} a$ value of the hydrate-based gas absorber was about 10 times higher than that of the bubble column, and therefore the hydrate-based gas absorber was better as a gas separator. However, the tendency of $K_{L} a$ may change due to differences in temperature and HFC134a mole fraction in feed gas between the hydrate-based gas absorber and the bubble column. We will consider the effects of the experimental conditions in further investigation.

\section{Acknowledgements}

A part of this research was funded by JSPS research grant $16 \mathrm{~K} 06821$.

\section{References}

Kavanaugh M. C. and R. R. Trussell; "Design of Aeration towers to Strip Volatile Contaminants from Drinking Water," J. Am. Water Works Assoc., 72, 684692 (1980)

Nagata T., H. Tajima, A. Yamasaki, F. Kiyono, and Y. Abe; "An analysis of Gas Separation Processes of HFC134a from Gaseous Mixtures with NitrogenComparison of Two Types of Gas Separation Methods, Liquefaction and Hydrate-Based Methods, in Terms of the Wquilibrium Recovery Ratio," Sep. Purif. Technol., 64, 351-356 (2009)

Shimizu, M., M. Hattori, K. Yamagiwa, and H.Tajima; "Mass Transfer Characteristics of Hydrate-Based Gas Separation Equipment," Proceedings of the 8th International Conference on Gas Hydrate, T4-35, Beijing, China (2014)

Sobieszuk P. and R. Pohorecki; "Gas-Side Mass Transfer Coefficients in a Falling Film Microreacter," Chem. Eng. Process., 49, 820-824 (2010)

Tajima, H., T. Nagata, Y. Abe, A. Yamasaki, F. Kiyono, and K. Yamagiwa; "HFC-134a Hydrate Formation Kinetics during Continuous Gas Hydrate Formation with a Kenics Static Mixer for Gas Separation," Ind. Eng. Chem. Res., 49, 2525-2532 (2010) 